\title{
Network overhead crowd management mechanism of virtual mobile Internet
}

\author{
Hai-yan $\mathrm{Wu}^{1 *}$, Wei-ping $\mathrm{Li}^{1,2}$ and Jin Wang
}

\begin{abstract}
It has become the hot research issue that solves the bottleneck of resource management in the development of Internet through virtualization. However, there are the challenges of mobility management, resource management, and network overhead management in the virtualization of the Internet. First, based on the mobile Internet network construction and management mode, the mobile Internet virtual model was proposed for managing the differences of the port communication between the mobile Internet protocol layer and protocol layer. Secondly, based on the network management cost control and the reconstruction of the Internet virtualization, we designed the network overhead crowd optimization space and management vector. The network overhead crowd management mechanism is proposed, which will transport the mobile virtualization Internet topology to point-to-point structure. Finally, the simulation results verified the advantages of the network overhead management mechanism of the virtual mobile Internet in terms of the cost and real time of the network overhead management.
\end{abstract}

Keywords: Network overhead, Crowd management, Virtual mobile Internet, Fully connected networks

\section{Introduction}

Network virtualization helps to improve the allocation of resources between Internet users and service providers [1], Internet users through the virtualization platform [2] resource registration, resource requests and resource response, and a series of operations. Virtualization network can improve the whole network connectivity [3] and improve the efficiency of resource scheduling [4]. Network virtualization has achieved a series of research results.

In article [5], a virtual network reconfiguration was proposed for data center networks that immediately reconfigure the virtual network so as to reduce the energy consumption under the constraints on the bandwidth and delay between servers in data center networks based on optical communication paths.

However, there are no further research of the influence of mobility, management cost, and complexity of virtual Internet of the above results. We proposed the network overhead crowd management mechanism based on virtual mobile Internet. An energy cost model was proposed by Sen $\mathrm{S}$ et al. [6], which could formulate the

\footnotetext{
* Correspondence: haihyanwu@sina.com

1 Department of Police Technology, Railway Police College, Zhengzhou 450053, China

Full list of author information is available at the end of the article
}

energy-aware virtual network embedding problem as an integer linear programming problem. An auxiliary graph model was discussed in article [7] to address multilayer virtual network mapping in a dynamic traffic scenario. The authors summarized and analyzed the Virtual Object as a Major Element of the Internet of Things [8]. The communicating while computing scheme was proposed in article [9] for the distributed mobile cloud computing over 5G heterogeneous networks. The author of article [10] completed the virtual labs project with the paradigm shift in an Internet-based remote experimentation. The SMART-hop setup request network was proposed, which is a specialized network that replaces long and overlapping broadcast wires with shorter wires and switches [11]. The article [12] proposed the use of the information embedded in an air tasking order during the planning phase of military missions to optimize the network performance. In article [13], the authors addressed the two-level dynamic scheme for improving the energy-efficient resource allocation and intercell-interference management of the heterogeneous networks. The priority of emergency vehicles at every intersection was given by designing a different set of local rules for expediting the response times [14]. The above articles ignored the management issue of virtual Internet. 
The comprehensive survey of Internet of things service definition, regulation, and standardization activities was given in article [15]. The article [16] discussed how to assign the crowdsourcing sensing tasks based on the credible interaction between users. The crowdsourceable framework was proposed to quantify the quality of experience of multimedia content [17]. There are no further research of the crowd management with the network overhead in the above articles.

The rest of the paper is organized as follows. Section 2 describes the mobile Internet virtual model. In Section 3, we discussed the network overhead crowd management mechanism. The performance analysis of network overhead crowd management mechanism has been shown in Section 4. Finally, the conclusions are given in Section 5.

\section{Mobile Internet virtual model}

There are many network construction and management mode in mobile Internet. In the mobile Internet, there is a difference between the different protocol layer and the protocol layer. In order to satisfy the coexistence needs of multiple modes and improve the system performance, the idea of network virtualization is introduced.
Network virtualization is embodied in the Internet mobility management, network formation, network resource management, network communication, etc. Mobile Internet virtual system is shown in Fig. 1. In order to enhance the consistency of the virtual mobile Internet and the actual Internet, the network routing update and building of the mobile Internet virtualization have to be enhanced. After the mobile Internet virtualization, the mobile Internet self-update and resource management model update should be always kept. In particular, the network virtualization can effectively solve the problem of the new network to join the Internet and the integration of new technical differences between complex networks by moving communication terminals and services. The mobile Internet resource management will be separated from the multilayer protocol cluster. Mobile Internet virtualization model includes network architecture virtualization, communication protocol virtualization, and virtualization of mobile services. Mobile Internet virtualization can provide a mobile Internet virtual platform between Internet service providers and mobile users. This platform helps to improve the performance of the mobile terminal in the frequent replacement of the interconnection between the subnetworks. This platform helps to satisfy the needs of large-scale user's concurrent access



Fig. 1 Virtual mobile Internet system 
services of the Internet and reduce the cost of large-scale mobile terminal deployment.

Mobile Internet resource management virtualization process is as follows:

Step 1, explore the mobile Internet resources.

Discovery results of the Internet resource

would be uploaded to the virtual management

center. Then the virtualization process is

waiting for the feedback.

Step 2, the virtualization management center

sends the Internet resource registration

information to the virtual platform.

Step 3, service provider sends a network

routing update request to the virtual

management center based on user resource

request information.

Step 4, the virtual management center sets

up a virtual subnetwork collection after

updating the network router.

Step 5, extract the valuable mobile Internet

resources.

Step 6, mobile Internet resources to build

the virtual module for satisfying the user

requirements. The resources section of this

module is consistent with the network resources

in the virtualization platform.

Mobile Internet virtualization costs are shown in the formula (1).

$$
\mathrm{VC}=\left\{\begin{array}{l}
\{N(i), T(i)\}\{M(j), \operatorname{SS}(i, j), \operatorname{SC}(j)\} \\
i=1,2 \ldots, n \\
j=i, i+1, \ldots, m \\
n<m
\end{array}\right.
$$

Here, VC is the cost of mobile Internet virtualization. $N$ represents the size of the virtual subnet. $T$ represents the time cost. $M$ represents the cost of mobility virtualization. SS represents the virtual cost of subnetwork switching. SC is the cost of virtualization Internet. Parameters $i$ and $j$ respectively represent two different mobile users. $n$ and $m$ respectively denote the size of the two different operators services.

\section{Network overhead crowd management mechanism}

Based on the network management cost control and the virtual network reconfiguration algorithm, we designed the network overhead crowd optimization space and the management vector. The overhead structure will convert the mobile virtualization Internet topology to the pointto-point architecture. Here, the network overhead of each protocol layer is defined as a crowd node. Internet access service is defined as a crowd resource request node. Each virtual module of the Internet virtualization platform is defined as a crowd management node. The management node is the center of the network overhead management mechanism. The crowd resource request node is connected to the node.

In order to further reduce the complexity of network overhead and management, every virtual crowd management node of the Internet virtualization platform must maintain a bidirectional connection with the crowd node. The connection must satisfy the mobility management of the randomness and the full connectivity of the Internet. Virtual mobile Internet virtual topology is the network overhead crowd management of the fully connected graph. The network overhead management driven by this kind of crowd is in exchange for the minimum interconnection cost with the virtual Internet mapping. This crowd management mechanism is based on the optimization of network cost as the goal, in order to solve the network overhead request of network overhead crowd optimization space, improve the speed of the virtual Internet restructuring, and reduce the complexity of network overhead management.

$N_{\mathrm{CP}}$ represents a crowd node. $N_{\mathrm{RCQ}}$ represents a resource request node. $N_{\mathrm{CM}}$ is a crowd management node. The value of $N_{\mathrm{CP}}$ is determined by the cross layer system of the virtualization platform. The value of $N_{\mathrm{RCQ}}$ is determined by the operator and the mobile user. The value of $N_{\mathrm{CM}}$ is determined by the virtual process of mobile Internet. NO $(\mathrm{a}, \mathrm{b})$ represents the network overhead between $\mathrm{a}$ and $\mathrm{b}$ nodes. NO $(\mathrm{a}, \mathrm{b})$ up represents the overhead network overhead between a and b nodes. NO $(\mathrm{a}, \mathrm{b})$ DW represents the network overhead between a and $b$ nodes.

$$
\left\{\begin{aligned}
N O(a, b)_{U P}= & \frac{\sum_{i=1}^{x} N O(i)}{N_{C P}}\left(\omega\left(N_{R C Q}\right)\right) \\
& \sum_{j=1}^{y} N O(j) \\
N O(a, b)_{D W}= & \frac{\sum_{i=1}^{N_{C P}} N O(i)}{} \\
& \sum_{j=1} N O(j)
\end{aligned}\right.
$$

Here, $x$ represents the uplink scale. $y$ indicates the size of the downlink. $\omega\left(N_{\mathrm{RCQ}}\right)$ denotes the average network overhead in the uplink virtualization network. $\omega_{F}\left(N_{\mathrm{RCQ}}\right)$ denotes the average network overhead in the downlink virtualization network.

The additional network overhead of crowd management node can be obtained by the formula (3). The 
added value of $V_{\text {ad }}$ can accurately reflect the fusion degree of crowd and network overhead management $F_{\mathrm{d}}$. The smaller the added value, the higher the degree of fusion.

$$
\left\{\begin{array}{l}
V_{a d}=\max \left\{\sum_{i \rightarrow x, j \rightarrow y} \omega\left(N O(i) \cdot N_{C M}(j)\right)\right\} \\
F_{d}=\ln \left(\frac{\omega(\min \{x, y\})}{\sum_{k \rightarrow N_{C P}} \omega_{F}(k)}\right)
\end{array}\right.
$$

According to the formulas (2) and (3), the implementation of network overhead crowd management process is shown in Fig. 2. The crowd mechanism must satisfy the conditions shown in the formula (4).

$$
\left\{\begin{array}{l}
\sum_{i=1}^{x} N O(i) \leq \sqrt{\omega(x)} \\
\sum_{i \rightarrow x, j \rightarrow y} \omega\left(i \cdot N_{C M}(j)\right) \geq \sum_{k \rightarrow N_{C P}} \omega_{F}(k)
\end{array}\right.
$$

\section{Performance analysis of network overhead group management algorithm}

Based on $C$ language, the proposed virtual mobile Internet platform was realized and simulated. In the experiment, the mobile Internet service platform is composed of randomly generated crowd node, crowd resource request node, and crowd management node. There are many concurrent links between mobile nodes and network overhead management centers. Mobile Internet physical network topology is virtualized. The resource request of mobile nodes and the network overhead request are initiated by the Poisson distribution. The time window of the Internet service initiated by each experiment is set to $1-5$. The network overhead management request of virtual Internet has to satisfy the requirements of equation (4). The average initiation time of the virtual Internet crowd requests is subject to exponential distribution. The resource request of the crowd points is subject to the uniform distribution. The experimental parameters are shown in Table 1 . We performed five experiments.

In the experiment, the delay of the link mapping shortest path management mechanism (LMSP) and one of the OCMVI (OCMVI: network overhead crowd management mechanism of virtual mobile Internet) mechanism are statistically shown in Fig. 3. Because the network virtualization process established in this paper accounted the mobility management of the Internet, network formation, network resource management, network communication, etc., at the same time, the consistency of the virtual mobile Internet and real internet was maintained by enhancing the mobile Internet virtualization network routing updates and building functions. In addition, the OCMVI mechanism has always maintained a mobile Internet self-update and resource management model update. Therefore, network virtualization of the OCMVI mechanism can effectively solve the access problem of heterogeneous network and the integration of new technical differences between



Fig. 2 Network overhead crowd management progress 
Table 1 Parameter settings

\begin{tabular}{lll}
\hline Step $(\mathrm{ms})$ & Starting time & End-to-end delay $(\mathrm{ms})$ \\
\hline 1 & $8: 0: 0$ & 0.08 \\
2 & $8: 1: 15$ & 0.19 \\
3 & $8: 10: 28$ & 0.09 \\
4 & $9: 0: 16$ & 0.2 \\
5 & $10: 23: 45$ & 0.3 \\
\hline
\end{tabular}

complex networks. The OCMVI mechanism has the excellent real-time performance. The OCMVI mechanism can effectively eliminate the delay jitter in the process of network virtualization and network overhead management.

Figure 4 shows the cost results of the OCMVI mechanism and the LMSP mechanism. From Fig. 4, the OCMVI mechanism has lower cost at different time and statistical steps. This is because the OCMVI mechanism designed the network overhead crowd optimization space and management vector. The overhead of crowd mechanism will convert virtual Internet topology to point-to-point structure. This mechanism can solve the problem of the crowd integration and network overhead management. The LMSP mechanism only considers the impact of network path on the network overhead and ignores the network overhead of the virtual process and the mapping of the virtual module.

\section{Conclusions}

Internet virtualization algorithm can effectively solve the problem of Internet resource management. However, the virtualization of the Internet has brought about the problems such as the mobility management, Internet resource management, and network overhead management. On the one hand, we researched the mobile Internet network construction and management. By constructing virtual mobile Internet platform, we solve the



Fig. 3 Delay of network overhead management

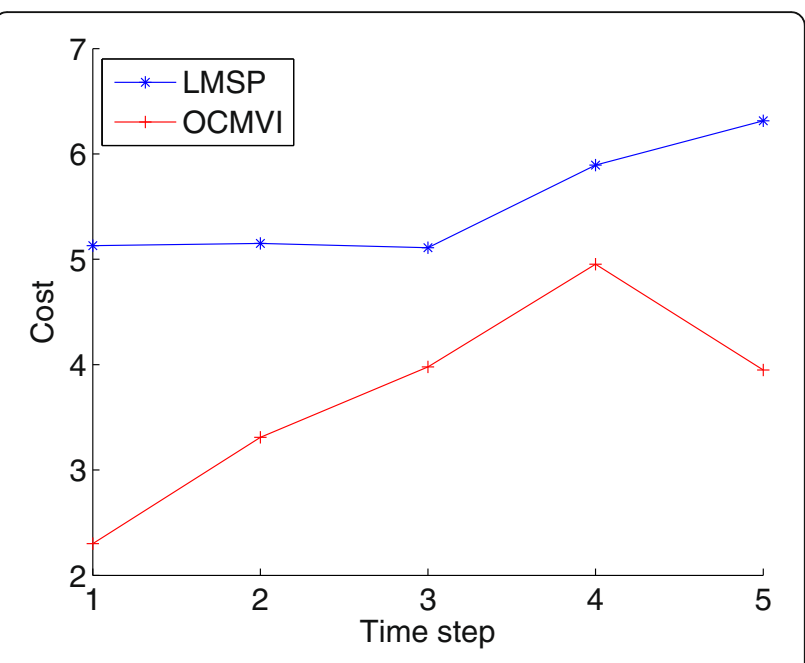

Fig. 4 Cost of network overhead management

mobile internet protocol communication differences between the internal layer and protocol layer problems. On the other hand, we designed the network overhead crowd optimization space and defined the management vector. The proposed crowd mechanism convert the mobile Internet topology to virtual point-to-point structure based on the combination of the network management cost and of virtual Internet reorganization control algorithm. The experimental results show that compared with the link mapping shortest path management mechanism, the proposed virtual mobile Internet network overhead crowd management mechanism can weaken jitter, shorten the delay, reduce the network overhead cost, and so on.

\section{Acknowledgements}

This work is supported in part by key scientific research projects of Henan Provincial Colleges and Universities No. 15A520092 and the basic research projects of the Central University in 2016 Nos. 2016TJJBKY022 and 2016TJJBKY007.

Competing interests

The authors declare that they have no competing interests.

\section{Author details}

${ }^{1}$ Department of Police Technology, Railway Police College, Zhengzhou 450053, China. ${ }^{2}$ Department of Information Engineering, School of Information Engineering, Wuhan University of Technology, Wuhan 430070, China.

Received: 22 July 2016 Accepted: 16 October 2016

Published online: 03 November 2016

\section{References}

1. MR Rahman, R Boutaba, SVNE: survivable virtual network embedding algorithms for network virtualization[J]. IEEE Trans. Netw. Serv. Manag. 10(2), 105-118 (2013)

2. B Song, MM Hassan, EN Huh, Delivering IPTV service over a virtual network: a study on virtual network topology[J]. J. Commun. Netw. 14(14), 319-335 (2012)

3. M Melo, S Sargento, U Killat et al., Optimal virtual network embedding: node-link formulation[J]. IEEE Trans. Netw. Serv. Manag. PP(4), 1-13 (2013) 
4. M Yoshinari, Y Ohsita, M Murata, Virtual network reconfiguration with adaptability to traffic changes[]]. J. Opt. Commun. Networking 6(6), 523-535 (2014)

5. Y Tarutani, Y Ohsita, M Murata, Virtual network reconfiguration for reducing energy consumption in optical data centers[]]. J. Opt. Commun. Networking 6(10), 925-942 (2014)

6. S Sen, Z Zhang, AX Liu, X Cheng et al., Energy-aware virtual network embedding[J]. IEEE/ACM Trans. Networking 22(5), 1607-1620 (2014)

7. J Zhang, Y Ji, M Song et al., Dynamic virtual network embedding over multilayer optical networks[J]. J. Opt. Commun. Networking 7(9), 918-927 (2015)

8. M Nitti, V Pilloni, G Colistra et al., The virtual object as a major element of the Internet of things: a survey[]]. IEEE Commun. Surv. Tutorials 18(2), 1-1 (2015)

9. S Barbarossa, S Sardellitti, PD Lorenzo, Communicating while computing: distributed mobile cloud computing over $5 \mathrm{G}$ heterogeneous networks[]]. IEEE Signal Process. Mag. 31(6), 45-55 (2014)

10. R Bose, Virtual labs project: a paradigm shift in internet-based remote experimentation[]]. IEEE Access 1, 718-725 (2013)

11. X Chen, NK Jha, Reducing wire and energy overheads of the SMART NoC using a setup request network[]]. IEEE Trans. Very Large Scale Integration Syst. 24(10), 3013-3026 (2016)

12. A Betances, KM Hopkinson, M Silvius, Context aware routing management architecture for airborne networks[J]. let Netw. 5(4), 85-92 (2016)

13. A Al-Zahrani, F Yu, An energy-efficient resource allocation and interference management scheme in green heterogeneous networks using game theory[]]. IEEE Trans. Veh. Technol. 65(7), 5384-5396 (2016)

14. OK Tonguz, W Viriyasitavat, A self-organizing network approach to priority management at intersections[]]. IEEE Commun. Mag. 54(6), 119-127 (2016)

15. A Meddeb, Internet of things standards: who stands out from the crowd?[J]. IEEE Commun. Mag. 54(7), 40-47 (2016)

16. J An, X Gui, Z Wang et al., A crowdsourcing assignment model based on mobile crowd sensing in the Internet of things[]]. IEEE Internet Things $J$, 2(5), 1-1 (2015)

17. CC Wu, KT Chen, YC Chang et al., Crowdsourcing multimedia QoE evaluation: a trusted framework[]]. IEEE Trans. Multimedia 15(5), 1121-1137 (2013)

\section{Submit your manuscript to a SpringerOpen ${ }^{\circ}$ journal and benefit from:}

- Convenient online submission

- Rigorous peer review

Immediate publication on acceptance

- Open access: articles freely available online

- High visibility within the field

- Retaining the copyright to your article

Submit your next manuscript at $\gg$ springeropen.com 